\title{
High-pressure neutron diffraction study of $\mathrm{Pd}_{3} \mathrm{Fe}$
}

\author{
Christopher J. Ridley, Craig L. Bull, Nicholas P. Funnell, \\ Silvia C. Capelli, Pascal Manuel, \\ Dmitry D. Khalyavin, Christopher D. O'Neill \\ and Konstantin V. Kamenev
}

\section{Published version information}

Citation: CJ Ridley et al. "High-pressure neutron diffraction study of $\mathrm{Pd}_{3} \mathrm{Fe}$." Journal of Applied Physics, vol. 125, no. 1 (2019): 015901.

DOI: $\underline{10.1063 / 1.5079804}$

This article may be downloaded for personal use only. Any other use requires prior permission of the author and AIP Publishing.

This version is made available in accordance with publisher policies. Please cite only the published version using the reference above. This is the citation assigned by the publisher at the time of issuing the AAM. Please check the publisher's website for any updates. 


\title{
High-pressure neutron diffraction study of $\mathrm{Pd}_{3} \mathrm{Fe}$
}

\author{
Christopher J. Ridley, ${ }^{1,}$ a) Craig L. Bull, ${ }^{1}$ Nicholas P. Funnell, ${ }^{1}$ Silvia C. Capelli, ${ }^{1}$ Pascal Manuel, ${ }^{1}$ Dmitry D. \\ Khalyavin, ${ }^{1}$ Christopher D. O'Neill, ${ }^{2}$ and Konstantin V. Kamenev ${ }^{3, \text { b) }}$ \\ 1) ISIS Neutron and Muon Source, Rutherford Appleton Laboratory, Chilton, Didcot OX11 OQX, \\ $U K$ \\ ${ }^{2)}$ The School of Physics and the Centre for Science at Extreme Conditions, The University of Edinburgh, Peter Guthrie Tait Road, \\ Edinburgh EH9 3FD, UK \\ ${ }^{3)}$ The School of Engineering and the Centre for Science at Extreme Conditions, The University of Edinburgh, \\ Peter Guthrie Tait Road, Edinburgh EH9 3FD, UK
}

(Dated: 29 November 2018)

High-pressure neutron diffraction data from powder and single-crystal samples of atomically disordered $(F m \overline{3} m)$ and ordered $(P m \overline{3} m) \mathrm{Pd}_{3} \mathrm{Fe}$ were collected up to pressures of $15 \mathrm{GPa}$, and high-pressure SQUID magnetometry data were collected up to $6 \mathrm{GPa}$. The data show a subtle decrease in the magnetic moment with applied pressure, resulting in a transition to a paramagnetic state by approximately $8 \mathrm{GPa}$ at $300 \mathrm{~K}$. Diffraction results have been used to determine the equation of state, resulting in a bulk modulus of 176.78(9) GPa for the disordered powder and 187.96(7) GPa for the ordered single-crystal samples respectively, approximately $20 \%$ more compressible than previously reported from X-ray measurements. High-temperature SQUID magnetometry was used to confirm the ambient pressure Curie temperature of the sample $(545 \mathrm{~K})$, which was further investigated using high-temperature single-crystal neutron diffraction at ambient pressure.

\section{INTRODUCTION}

Pd-Fe alloys are of interest as they demonstrate a number of unusual properties due to the interplay between structural and magnetic characteristics. For example, magnetic shape memory (high strain induced by a magnetic field) is observed in face-centred tetragonal (fct) Fe-rich alloys $(30 \% \mathrm{Pd})$ of the series $^{1,2}$, and near-zero thermal expansion $\left(\alpha=8.5 \times 10^{-6}\right.$, between $273-313 \mathrm{~K}$ ) is observed in disordered alloys with approximately $30 \% \mathrm{Pd}$ at ambient pressure around room temperature (hereby referred to as 'Invar behaviour', after the anomaly first observed in $\left.\mathrm{Fe}_{65} \mathrm{Ni}_{35}\right)^{3-5}$. More recently a pressure induced Invar state was reported in powder samples of $75 \%$ Pd alloys ${ }^{6}$. As such, these alloys provide an excellent platform for comparison with physical models of transition metal systems, 3d-electronic structures, and magnetism in binary compounds.

The structural phase diagram of the $\mathrm{Pd}_{x} \mathrm{Fe}_{(1-x)}$ system may be summarised as follows; in the range $0 \leq x \leq 0.28$ the alloy is in the $b c c$ phase $(\alpha)^{7}$, transforming to a pure $f c t$ phase $\left(\gamma^{\prime}\right)$ by $x \approx 0.5$. With further increasing Pd content a pure $f c c$ phase $(\gamma)$ is formed by $x \approx 0.7$, though there are slightly differing reports as to the exact composition of transformation ${ }^{8,9}$. At $x=0.75, \mathrm{Pd}_{3} \mathrm{Fe}$ forms the expected $f c c$ phase with a mixture of $\mathrm{Fe}$ and $\mathrm{Pd}$ throughout the unit cell. This is then atomically site-ordered through annealing at $875 \mathrm{~K}$, resulting in a change of symmetry. The ordered phase has space group $P m \overline{3} m$, with Fe on sites of type 1(a) $(0,0,0)$ and Pd on 3(c) $(0,0.5,0.5)$. Whereas the disordered phase has symmetry $F m \overline{3} m$, with $\mathrm{Fe}$ and Pd partially occupying sites of type $4(a)(0,0,0)$. Both the atomically ordered, and disordered phases are ferromagnetically ordered; the latter showing $\mathrm{a} \approx 15 \mathrm{~K}$ lower Curie transition temperature ${ }^{10}$.

\footnotetext{
a) christopher.ridley@stfc.ac.uk

b)k.kamenev@ed.ac.uk
}

A number of theories have been proposed to explain the Invar phenomenon (see discussion in Wasserman ${ }^{11}$, Lagarec et al. ${ }^{12}$ and references within), though the most long standing theory was proposed by Weiss ${ }^{13}$. Weiss explained this behaviour using a two-state model $\left(\gamma_{1}, \gamma_{2}\right)$, where $\gamma_{1}$ was associated with a high-moment ferromagnetic, large volume state, and $\gamma_{2}$ with a low-moment antiferromagnetic, reduced volume state. Through a thermal depopulation of $\gamma_{1}$, and population of $\gamma_{2}$, this model is quite successful at reproducing experimental findings. However, experimentally there is no evidence for the coexistence of two states in the region of this transition, suggesting that the model is purely phenomenological ${ }^{14}$. Furthermore, where more realistic non-collinear or partial antiferromagnetic structures are considered, it is found that the double energy minimum becomes a single minima, thus breaking the model ${ }^{15}$. An alternative disordered local-moment model describes Invar materials as high magnetic-moment structures, driven to local moment (order-disorder) orientation through strong exchange between Fe sites, which drives the continuous volume collapse ${ }^{16-18}$.

The use of pressure to tune the Invar transition provides a unique tool to understand the transition, and subsequently the mechanism for zero thermal expansion in these materials. Pressure-induced Invar behaviour was first demonstrated in an Fe-Ni alloy ${ }^{19} ; \mathrm{Fe}_{0.55} \mathrm{Ni}_{0.45}$ was shown to have a near-zero coefficient of thermal expansion at 7.7 GPa from $291-500 \mathrm{~K}$.

This study is focussed on the $\mathrm{Pd}_{3} \mathrm{Fe}$ alloy which is ferromagnetic at ambient conditions $\left(\mathrm{T}_{c} \approx 540 \mathrm{~K}^{20}\right)$, and is reported to display high-pressure Invar behaviour at room temperature. $\mathrm{Pd}_{3} \mathrm{Fe}$ reportedly undergoes a large volume collapse of $\Delta \mathrm{V} \approx 5 \%$ between $9-14 \mathrm{GPa}$ as measured with energy dispersive $\mathrm{X}$-ray diffraction, with an apparent collapse of long-range magnetic order, previously observed through Mössbauer spectroscopy ${ }^{6}$. Near-zero thermal expansion over the range $300-523 \mathrm{~K}$ is also reported, before the Curie temperature is reached ${ }^{21}$. Density functional calculations have not conclusively characterised what magnetic state the sam- 
ple is in post-collapse $\mathrm{e}^{22}$. More recent theoretical studies have suggested that the alloy forms a triple-Q state where the moments on the lattice alternate pointing toward and away from the centre of the lattice ${ }^{23}$. This would support the theory of spin frustration (non-collinear magnetism), and it is speculated that a pressure-driven reduction of the Curie temperature could trigger this order-disorder transition, which in turn drives the Invar behaviour of $\mathrm{Pd}_{3} \mathrm{Fe}^{22}$.

At ambient conditions the sample is a simple ferromagnet, though a number of reports in the literature have suggested that the sample forms a non-collinear magnetic state when driven through the volume collapse at high pressure. The aim of this study was to investigate the magnetic state of atomically ordered $\mathrm{Pd}_{3} \mathrm{Fe}$ as a function of pressure, to further understand the previously reported high pressure state, and the mechanism behind its Invar behaviour. Neutron diffraction measurements as a function of pressure should reveal the loss in long range magnetic order, and allow the quantification of the drop in measured moment. Powder neutron diffraction, and magnetometry measurements were performed to support these data.

\section{SAMPLE PREPARATION}

The samples were prepared from compacted pellets of Pd (99.999\%, Sigma Aldrich) and $\mathrm{Fe}(99.998 \%$, Alfa Aesar) powder. Four sample melts of approximately $5 \mathrm{~g}$ were prepared. For two of the batches, the Fe pellets were first annealed in a flowing atmosphere of $\mathrm{H}_{2}$ : $\mathrm{Ar}$ up to $755 \mathrm{~K}$ to remove any oxide content, this was confirmed through measuring released $\mathrm{H}_{2} \mathrm{O}$ content via mass spectrometry, and measuring the mass loss post annealing. For all batches the $\mathrm{Fe}$ was annealed in ultra-high vacuum (UHV) to break down any remaining hydroxide and water content in the pellet. For all batches the Pd was annealed in UHV at $1175 \mathrm{~K}$ to reduce hydrogen and water content. All samples of the alloy were prepared by induction melting stoichiometric quantities of the pellets in a $\mathrm{Cu}$ crucible, forming a boule of the alloy. In all cases the precursor pellets were observed to melt together at $1095 \mathrm{~K}$, in agreement with literature values ${ }^{24,25}$. To improve homogeneity in the sample, it was allowed to remain molten for approximately 1 hour before cooling. This was performed in a pressurised ultra-pure argon atmosphere to prevent significant mass loss due to vapour pressure, reducing the risk of compositional changes during this period. As the copper crucible is actively cooled, the risk of contamination from the container is minimal. No excessive change in the total mass was measured during this process. Quenching the melt from high temperature resulted in the disordered phase of the alloy; to achieve the atomically ordered face-centred cubic phase $(P m \overline{3} m)$ the samples were annealed for 9 days at $875 \mathrm{~K}$ in UHV, and cooled slowly back to room temperature ${ }^{26}$. The level of order/disorder was determined using powder X-ray diffraction. Single-crystals were grown using the Czochralski method, with crystals pulled from the melt using a rotating tungsten needle ${ }^{27}$. The resulting growth was grain mapped using X-ray Laue diffraction ${ }^{28}$, and a single-crystal was iso- lated, orientated, and electrical discharge machined (EDM) using a fine, high purity, $\mathrm{Cu}$ wire under paraffin oil. The samples were then re-annealed to ensure full site ordering. To give an initial indication of the composition of the final samples, Xray fluorescence measurements were performed (PANalytical Epsilon3 XL), which determined a Pd content between 73\% and $77 \%$ with repeated measurements on the same sample resulting in fluctuations around the expected $75 \%$ composition, demonstrating the limited accuracy of measurement.

\section{EXPERIMENTAL DETAILS}

All neutron diffraction measurements were performed at the ISIS Neutron and Muon Source. The time-of-flight (t.o.f.) Laue diffraction technique was used on the SXD diffractometer ${ }^{29}$ at $295 \mathrm{~K}$ and $555 \mathrm{~K}$ on an atomically ordered single-crystal with dimensions of approximately $0.5 \times 0.8 \times$ $1 \mathrm{~mm}^{3}$. Data were collected in a series of five orientations for each temperature; the data collection time for each orientation was $3 \mathrm{~h}$ and $5 \mathrm{~h}$ for each temperature respectively. Cell dimensions and Bragg intensities were extracted using the three-dimensional profile-fitting method implemented in the SXD2001 software ${ }^{30,31}$ for a total of 525 (70 unique, $\mathrm{R}_{\sigma}=0.066$ ) and 355 (50 unique, $\mathrm{R}_{\sigma}=0.048$ ) wavelengthdependent reflections respectively at $295 \mathrm{~K}$ and $555 \mathrm{~K}$. Data were corrected for the Lorentz effect but no absorption correction was applied. Both structures were refined by full matrix least squares on $\mathrm{F}^{2}$ using SHELX software ${ }^{32}$ in the $P m \overline{3} m$ space group using 6 parameters, the final $\mathrm{R}$ factors were 0.064 and 0.098 and goodness-of-fit parameters were 1.09 and 1.34 at $295 \mathrm{~K}$ and $555 \mathrm{~K}$ respectively. There was no evidence for partial disorder in the crystal. No attempt was made to refine the magnetic structure at room temperature.

Single-crystal t.o.f. neutron diffraction measurements were performed at high-pressure using a VX4 Paris-Edinburgh press on the WISH diffractometer ${ }^{33}$, and high-pressure neutron powder-diffraction measurement were performed using a V4 Paris-Edinburgh press on the PEARL diffractometer ${ }^{34}$. The samples were loaded using double-toroidal sintereddiamond anvils, and a null-scattering modified encapsulated TiZr gasket ${ }^{35}$. Fully deuterated methanol:ethanol in a 4:1 volume ratio was used as a pressure transmitting medium, remaining fully hydrostatic up to $10.5 \mathrm{GPa}$. In order to reach pressures above this hydrostatically, the sample was heated following the melting line of the pressure medium, as detailed elsewhere ${ }^{36}$. Sample pressure was determined using the equation of state of $\mathrm{Pb}^{37}$. Alignment of the single-crystal was maintained in the cell through mounting the cut crystal on a half filled gasket ${ }^{38}$.

High-temperature magnetometry was performed using a Quantum Design MPMS 3 VSM with furnace attachment. High-pressure magnetometry was performed using a Quantum Design MPMS XL. A small piece of sample was loaded into a turnbuckle diamond anvil cell ${ }^{39}$ with methanol:ethanol 4:1 as a pressure medium, and a ruby sphere used as a pressure marker ${ }^{40}$. Moment-temperature sweeps were performed in an external field of $20 \mathrm{mT}$ in the temperature range $2-600 \mathrm{~K}$. 
High-pressure magnetometry measurements were performed by performing field-sweeps at ambient temperature. The sample was cycled in field, and measurements were performed on field increase. The maximum field was chosen as $800 \mathrm{mT}$ to ensure sample saturation.

\section{RESULTS AND DISCUSSION}

\section{A. Single-crystal neutron diffraction: high pressure}

The $\pm 7^{\circ}$ out of plane aperture of the anvils from the pressure cell, and the cubic symmetry of the sample, allowed for the measurement of one set of reflections from the sample within the cell. The crystal was cut with $\{h 00\}$ in plane, with this set of reflections scattering in the $90^{\circ}$ geometry, providing a good balance between magnetic contribution to the measured signal and instrumental resolution. The data were normalised against incident beam current, but were uncorrected for attenuation from the gasket material, allowing only for a comparison of the relative intensities of the reflections.

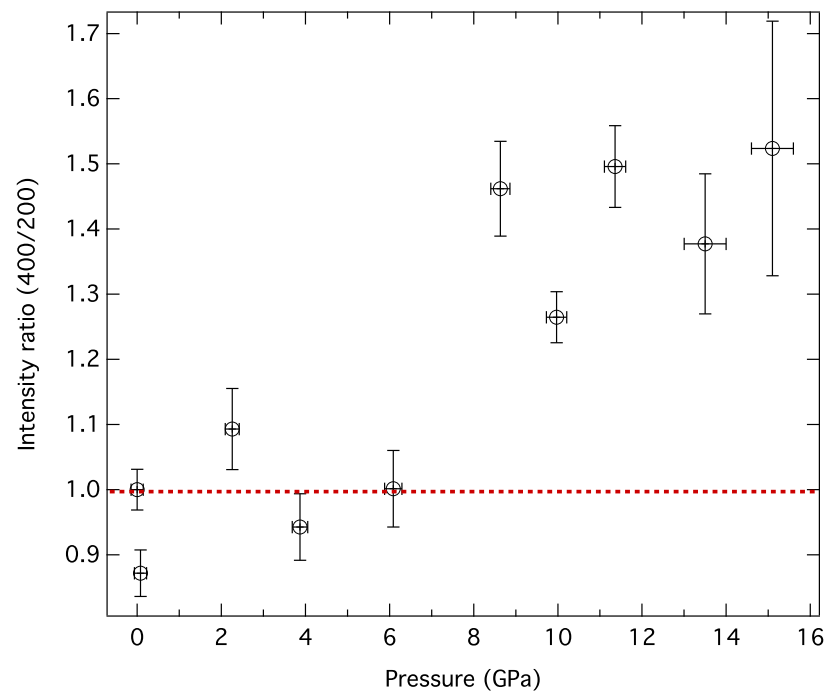

FIG. 1. Ratio of the integrated intensities above background of the $(400) /(200)$ reflections as a function of pressure. The values have been normalised to the value at ambient pressure. Relative changes in the intensities of the two reflections are indicative of changes in the magnetic moment in the sample, due to the fixed orientation of the crystal. As the (400) reflection becomes relatively more intense, this suggests a loss of long range order in the region $6-8 \mathrm{GPa}$. The error bars have been calculated based on the propagated uncertainties of the integrated intensities.

With a fixed scattering angle, the relative intensities of the $\{h 00\}$ reflections above the background at each pressure were considered to give a measure of the change in magnetic moment, due the magnetic form factor (see Figure 1). The data show almost no change in moment until $6-8 \mathrm{GPa}$ where a discontinuity is observed, indicating a drop in the magnitude of the magnetic contribution to these reflections. No further discontinuities were observed outside of error up to the max- imum pressure of $15 \mathrm{GPa}$. The cell was heated at pressures above $10 \mathrm{GPa}$ to retain hydrostaticity, to a maximum of $360 \mathrm{~K}$; as such this transition is known to be purely an effect of applied pressure. The apparent discontinuous nature of the transition is perhaps misleading, due to the limited number data points around the transition, and due to the large uncertainties on the integrated intensities. This is due to the (400) reflection being significantly weaker than the (200) reflection measured from the pressure cell (see SI). This aside, this relative change in integrated intensity is indicative of a loss of moment, with the sample forming either a low-spin state, or simply becoming paramagnetic at elevated pressure. The latter being generally consistent with existing magnetometry measurements reported elsewhere for disordered $\mathrm{Fe}-\mathrm{Pd}$ alloys ${ }^{41}$, and for $\mathrm{Fe}$ $\mathrm{Ni}$ Invar alloys ${ }^{42}$. Due to the number of data points around the transition pressure, it is not entirely clear whether the nature of the transition is first or second order. Nuclear forward scattering data from Winterrose et al. ${ }^{6}$ show no clear changes in the time modulation approaching the transition, which is suggestive of a first-order transition to a non-paramagnetic state. The present data don't eliminate the possibility of a transition to a non-paramagnetic state with a lower ordering temperature. However, no structural changes or anomalies are observed under compression at the temperatures considered.

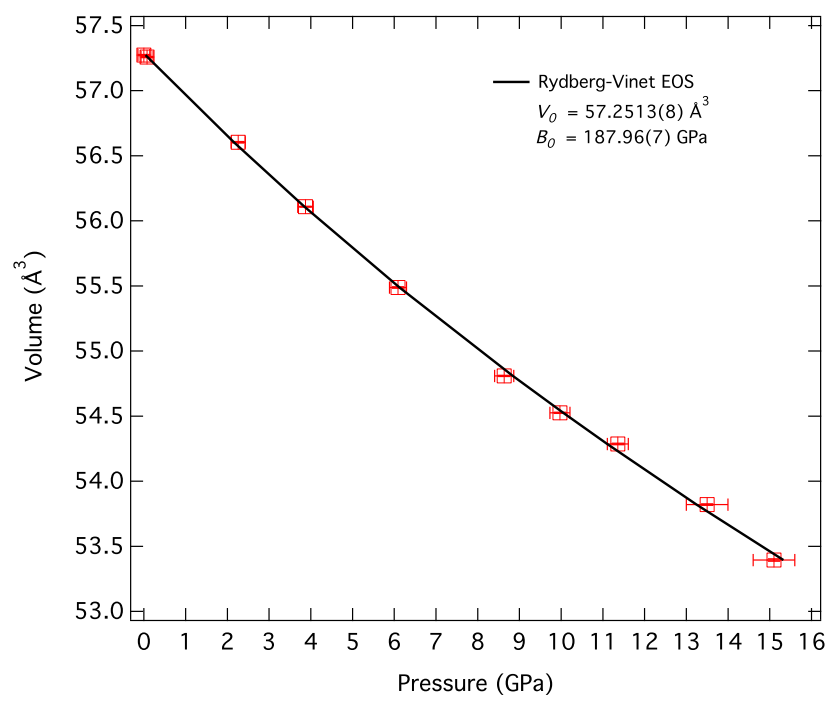

FIG. 2. Isothermal compressibility of the single-crystal sample determined from a Rydberg-Vinet equation of state.

The isothermal bulk modulus of the sample was determined through fitting the measured unit cell volume to a RydbergVinet equation of $\operatorname{state}^{43}$, see Figure 2. The fit parameters are summarised in Table II, and discussed in section IV B. The single-crystal study did not show any transition to a lowvolume state in the range $10-15 \mathrm{GPa}$, expected to occur begin by $9 \mathrm{GPa}$. No evidence for further magnetic transitions is seen, with no further discontinuities seen in the relative peak intensities. The recovered crystal showed no sign of damage or plastic deformation after compression, suggesting that very good hydrostatic conditions were maintained throughout the loading. 
To further investigate this change in the relative intensities of the (400) and (200) reflections, the sample was measured at ambient temperature and $555 \mathrm{~K}$ at ambient pressure on the SXD instrument. $555 \mathrm{~K}$ was chosen so as to be nominally above the previously reported Curie transition temperature of $540 \mathrm{~K}^{20}$. The measured, and refined intensities of these reflections are shown in Table I. These show that a) the absolute value of the intensities of both reflections decreases at high temperature, and b) that the ratio $(400) /(200)$ increases to approximately 1.3 times the ambient temperature value. This is consistent with a loss of magnetic ordering in the sample as expected at this temperature. The magnitude of the change in the signal is comparable to that measured during the high pressure experiment, within error when the different orientations of the crystal are considered between the two experiments, suggesting that the sample become paramagnetic under pressure, rather than forming a low-spin state.

TABLE I. Summary of calculated and observed intensity values for the (200) and (400) reflections observed during measurements on $\mathrm{SXD}$ at room temperature and $555 \mathrm{~K}$ above the Curie temperature. Measured wavelength, and calculated intensity ratios are also shown.

\begin{tabular}{|c|c|c|c|c|c|}
\hline $\mathrm{hkl}$ & $\mathrm{F}_{c}$ & $\mathrm{~F}_{o}$ & $\sigma\left(\mathrm{F}_{o}\right)$ & $\lambda(\AA)$ & Ratio $(040) /(020)$ \\
\hline $0 \overline{2} 0$ & 1464.08 & 1755.30 & 11.50 & 1.13 & $0.66(3)$ \\
\hline $0 \overline{4} 0$ & 1163.41 & 1166.36 & 46.76 & 0.56 & \\
\hline $0 \overline{2} 0$ & 1464.08 & 1654.12 & 15.06 & 0.89 & \\
\hline 020 & 1464.08 & 1551.41 & 10.68 & 1.12 & $0.70(3)$ \\
\hline 040 & 1163.41 & 1027.15 & 44.04 & 0.56 & \\
\hline \multicolumn{6}{|c|}{$555 \mathrm{~K}$} \\
\hline hkl & $\mathrm{F}_{c}$ & $\mathrm{~F}_{o}$ & $\sigma\left(\mathrm{F}_{o}\right)$ & $\lambda(\AA)$ & Ratio $(040) /(020)$ \\
\hline $0 \overline{2} 0$ & 1738.98 & 1475.59 & 12.55 & 1.82 & $0.83(2)$ \\
\hline $0 \overline{4} 0$ & 1217.25 & 1230.70 & 29.66 & 0.91 & \\
\hline 020 & 1738.98 & 2114.62 & 36.14 & 3.68 & $0.81(2)$ \\
\hline 040 & 1217.25 & 1711.98 & 23.62 & 1.85 & \\
\hline
\end{tabular}

\section{B. Powder neutron diffraction: effect of ordering}

Since the volume determination of the single-crystal was based on the measurement of a very limited number of reflections, the compressibility of the sample was reinvestigated using polycrystalline samples in both ordered and disordered states. Initially the two samples were characterised at ambient pressure in a vanadium container. While a laboratory $\mathrm{X}$-ray source was sufficient to nominally determine the ordering of the sample, neutron diffraction provided the added advantage of probing a larger bulk of material, allowing the as-synthesised sample boule to be characterised without the effects of strain induced from cutting or grinding the sample.
This is particularly important as previous reports have suggested that mechanical grinding may also induce disorder in these samples ${ }^{44}$. The larger sample volume used in pressure cells for neutron diffraction studies ${ }^{45}$ also allow for a more representative diffraction pattern than can be obtained from a focussed X-ray beam in a diamond anvil cell.

Figure 3 shows the patterns from the ordered and disordered boules in a vanadium sample container. The data are intensity extracted with a LeBail fit. A full Rietveld refinement was attempted, however, the self-attenuation of the sample could not be satisfactorily corrected for. The ordered sample shows the expected difference in peak intensities, and the appearance of the mixed $(h k l)$ even and odd reflections which are systematically absent from the $F$-centred disordered sample.

TABLE II. Summary of the equation of state data determined from the present work, compared with previously measured or calculated values from the literature. The value for $\mathrm{B}_{0}$ ' was fixed at a value of 4 , as discussed in the text.

\begin{tabular}{cccc}
\hline \hline Source & $V_{0}\left(\AA^{3}\right)$ & $B_{0}(\mathrm{GPa})$ & $B^{\prime}$ \\
\hline Single-crystal (exp) & $57.2513(8)$ & $187.96(7)$ & 4.0 (fixed) \\
Single-crystal (exp) & $57.29(33)$ & $173(9)$ & $6(2)$ \\
Ordered powder (exp) & $57.23(14)$ & $183(1)$ & 4.0 (fixed) \\
Ordered powder (exp) & $57.2812(9)$ & $164.9(2)$ & $8.36(4)$ \\
Disordered powder (exp) & $57.24(5)$ & $176.78(9)$ & 4.0 (fixed) \\
Disordered powder (exp) & $57.27(4)$ & $162(2)$ & $9.5(7)$ \\
Winterrose et al. $^{6}$ (exp) & $54.5(2)$ & $229(2)$ & 4.0 (fixed) \\
Kuhnen and Da Silva ${ }^{46}$ (calc) & & 180 & \\
De Jong et al. ${ }^{47}$ (calc) & 59.1 & 174 \\
\hline \hline
\end{tabular}

It was found that the peaks associated with the ordering of the structure have a broader peak-shape than those from the parent structure. The data were fitted using an $(h k l)$-selective size broadening model in FullProf ${ }^{48}$. While the intensity of these reflections suggest close to complete ordering in the sample, selective peak broadening represents a level of local disorder in the $5 \mathrm{~g}$ sample boule, where some Pd is still found on the $(0,0,0)$ site. No evidence of selective peak broadening was seen in the single-crystal data presented here, though it has been reported elsewhere for the structurally similar alloys $\mathrm{Cu}_{3} \mathrm{Au}^{49}$, and $\mathrm{Ni}_{3} \mathrm{Al}^{50}$.

To investigate the compressibility of the sample, and the discrepancy with previously measured values observed in the single-crystal data, both the ordered and disordered polycrystalline samples were compressed. As no volume collapse was expected in the disordered sample, this was only compressed up to approximately $6 \mathrm{GPa}$, while the ordered sample was again compressed up to approximately $15 \mathrm{GPa}$ (see SI for representative high pressure diffraction pattern). Figure 4 shows the measured equations of state for both samples, with the fit parameters summarised in Table II. The sample was heated above $10 \mathrm{GPa}$ in order to follow the melt- 

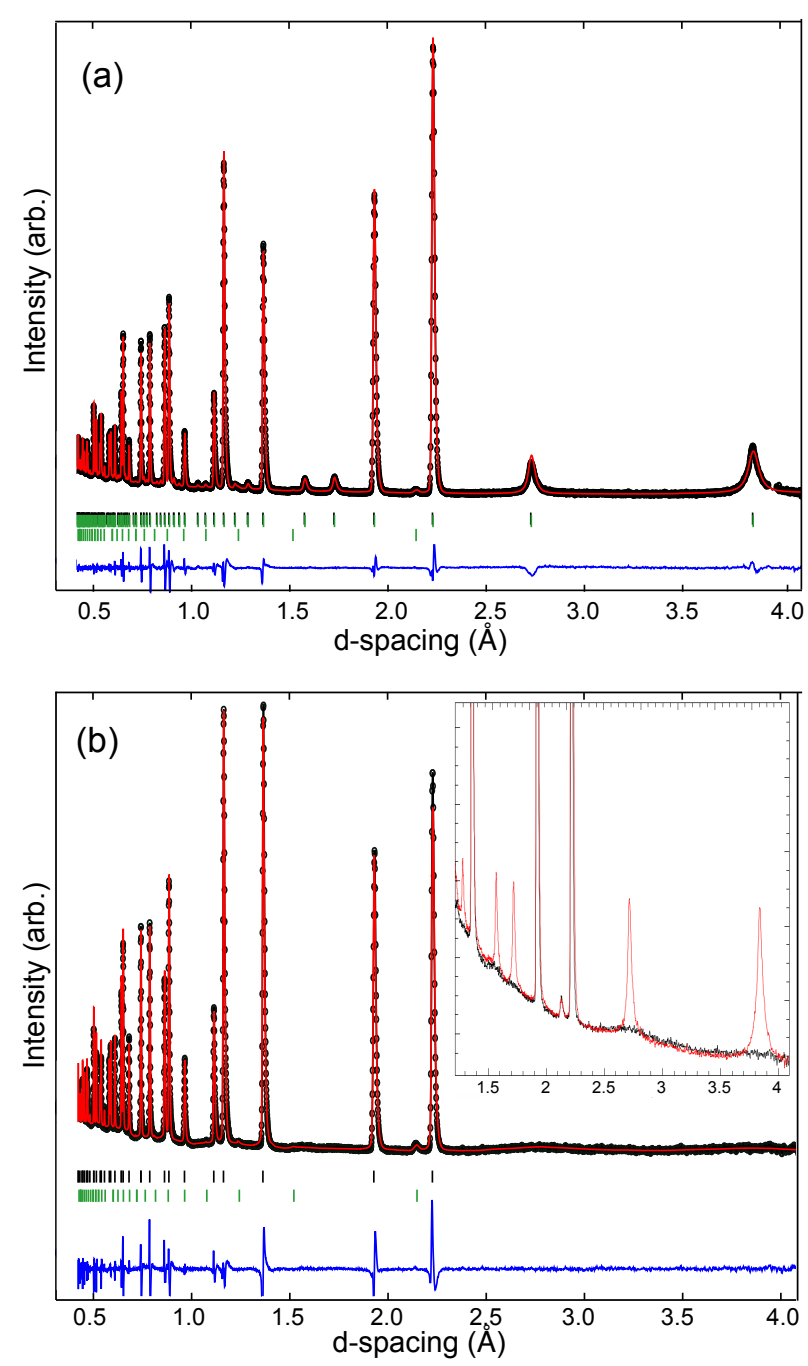

FIG. 3. Powder time-of-flight neutron diffraction data from PEARL collected from as-prepared boules of $\mathrm{Pd}_{3} \mathrm{Fe}$ in a $15 \mathrm{~mm}$ vanadium can at ambient temperature and pressure. In all plots the data are LeBail fitted (discussed in the text), the upper tick marks are for the sample, and the lower account for a small contribution from the vanadium container. No additional peaks were identified in the data. The LeBail fit is shown (red line) along with the measured data (black markers). (a) Ordered $P$-centred sample, annealed at $600{ }^{\circ} \mathrm{C}$ for 9 days. (b) Disordered $F$-centred (fcc) sample as quenched from the melt. (inset) expanded region showing appearance of systematically absent mixed $(h k l)$ even and odd indexed peaks. Note the difference in peak widths, also discussed in the text.

ing line of methanol:ethanol (4:1) to preserve hydrostaticity. The volume data from the sample were corrected accordingly using the measured thermal expansion coefficients as determined by Masumoto, Saitô, and Kobayashi ${ }^{3}$. At the highest temperatures achieved it was found that the shift in volume due to thermal expansion was no larger than $0.2 \%$, significantly smaller in magnitude than any expected volume collapse. The ordered sample was found to be approximately $5 \%$ less compressible than the disordered sample, which is consistent with calculations in similar materials ${ }^{51}$, and with the magnetic measurements of these samples discussed in section IV C. This difference in compressibility between the ordered and disordered state is related to the magnetostrictive properties of the materials ${ }^{52}$, due to the differing strains involved in the reorientation of magnetic domains in the sample ${ }^{51}$.

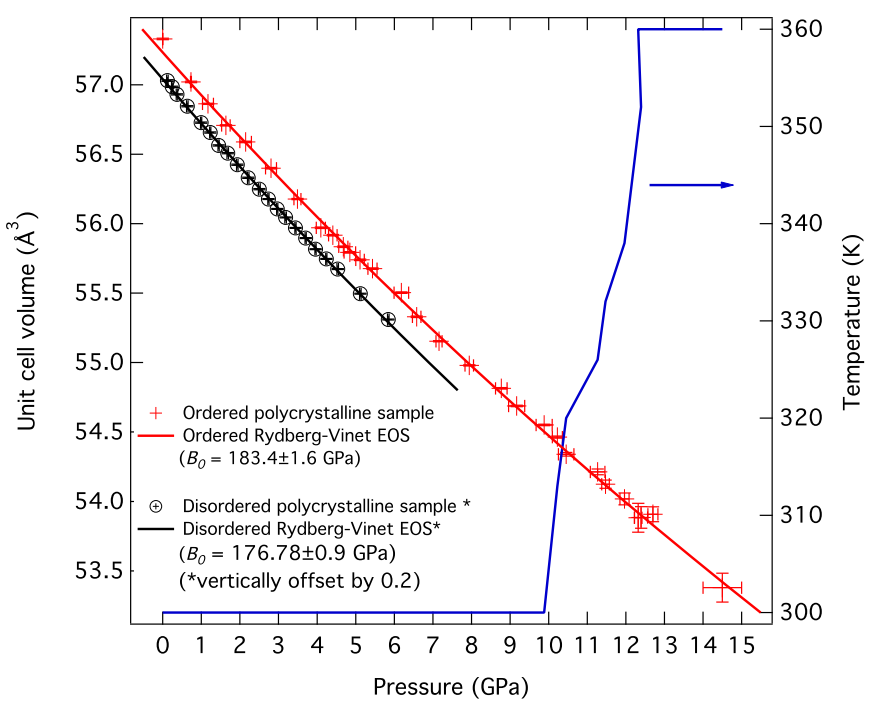

FIG. 4. (left) Compressibility of ordered and disordered polycrystalline samples as measured on PEARL, shown with Rydberg-Vinet equations of state for ordered sample up to $14.5 \mathrm{GPa}$, and disordered sample up to $5.8 \mathrm{GPa}$. The data and fit for the disordered sample have been vertically offset for clarity. The error bars on the pressures are estimated from the equation of state,. Both unit cell volume and pressure error bars are partially obscured by the size of the markers. (right) Temperature of the sample at each pressure, increased above $10 \mathrm{GPa}$ to maintain hydrostaticity of methanol:ethanol pressure medium. The volumes at pressures above $10 \mathrm{GPa}$ have been corrected for thermal expansion, as discussed in the text.

The measured value of $B_{0}$, with $B^{\prime}=4$ fixed, was found to be approximately $20 \%$ lower than that reported by Winterrose et al. ${ }^{6}$, whilst remaining highly consistent throughout the measurements reported in this study, but is significantly closer to the values determined by DFT calculations in the literature by Kuhnen and Da Silva ${ }^{46}$, De Jong et al. ${ }^{47}$. In addition, the value of the bulk modulus measured in this study is closer to that expected from comparison to the electronically similar $\mathrm{Pd}_{3} \mathrm{Mn}^{53}$, and structurally similar $\mathrm{Ni}_{3} \mathrm{Fe}^{51,54}$. The reason for the absence of the previously reported large volume collapse is unclear, though this may be due to the improved hydrostaticity of the sample conditions in the present study. Methanol:ethanol (4:1 by volume) was used rather than silicone oil, which has been reported to have an extremely low hydrostatic limit of $0.9 \mathrm{GPa}^{55}$, with unpredictable behaviour perhaps linked to the grade of silicone oil used ${ }^{36}$, and a possible phase transition observed in silicone oil between $12-15 \mathrm{GPa}^{56}$. Very similar discrepancies in the measured compressibilities between silicone oil and argon loadings are reported in the literature for an unrelated material ${ }^{57}$, which is attributed to differing hydrostaticity. 


\section{Magnetometry measurements}

The same ordered single-crystal used for the neutron measurements on WISH was characterised through magnetometry over a temperature range $5-600 \mathrm{~K}$. The sample was zero-field cooled and then warmed in a field of $20 \mathrm{mT}$. The moment-temperature curve of the sample is shown over the range $480-600 \mathrm{~K}$ in Figure 5. The high temperature susceptibility was fit to a linear Curie-Weiss law, $1 / \chi=(T-\Theta) / C$, where $\Theta$ is the paramagnetic Curie-Weiss temperature and $\mathrm{C}$ is the Curie constant. $\Theta$ was determined to be $550 \mathrm{~K}$, whereas the Curie temperature of the transition $T_{C}$, as determined by the minimum in $d M / d T$ vs. $\mathrm{T}$, is closer to $545 \mathrm{~K}$. Both of these results are in reasonable $(1 \%)$ agreement with literature values $^{21}$. No evidence for any further transitions was found over the temperature range considered.

Moment field sweeps were performed at a series of temperatures from $5-305 \mathrm{~K}$. As the moment doesn't entirely saturate at the highest fields considered, the saturated magnetic moment, $\mathbf{M}_{s}$ is extracted through fitting the high field approach to saturation with the empirical relationship:

$$
M=M_{s}\left(1-a / H-b / H^{2}-\ldots\right)+\chi_{0} H
$$

Here $a, b$, and $\chi_{0}$ are constants at each temperature, where the $\chi_{0}$ term is an additional susceptibility term included to account for high field induced band splitting. These are shown in insets of Figure 6. This shows that an expected decrease in saturated moment as a function of increasing temperature, following a Curie-Weiss law. The determined saturated moment at room temperature of $0.963(8) \mu_{b}$ /atom is in excellent agreement falling between the values determined by Fallot ${ }^{21}$ and Crangle ${ }^{58}$, showing that the sample is of similar quality between these studies.

The lower inset of Figure 6 shows the saturated magnetic moment as a function of temperature below room temperature, as determined from the moment-field measurements at each temperature. The data were fitted to the function $M_{S}=M_{0}(1-$ $c T^{\beta}$ ), where $M_{0}$ is the saturated moment at zero temperature and $c$ is a material constant, and $\beta$ is set to either $3 / 2$ or 2 depending on the model considered. It was found that a $\beta=$ 2 expression fitted the data slightly better than a Bloch $\beta=$ $3 / 2$ model, over the temperature range $5-190 \mathrm{~K}$. Although there are too few data points to to completely disregard the Bloch function, the slight improvement in fit is consistent with findings published elsewhere ${ }^{59}$.

To further investigate a possible ferro- to paramagnetic transition in the sample, high-pressure moment-field measurements were performed at ambient temperature; these are shown in Figure 7. The sample was cycled in magnetic field, and measurements were performed on increasing field, to give a measure of changes in sample saturation. With increased pressure up to $6 \mathrm{GPa}$ the changes are subtle. The saturated magnetic moment is observed to decrease by approximately $8.5 \%$, whereas the profile of the $\mathrm{MH}$ curve clearly flattens, tending towards a more paramagnetic-like signal. This change in moment is in agreement with the single-crystal neutron data, within the error of the measurements.

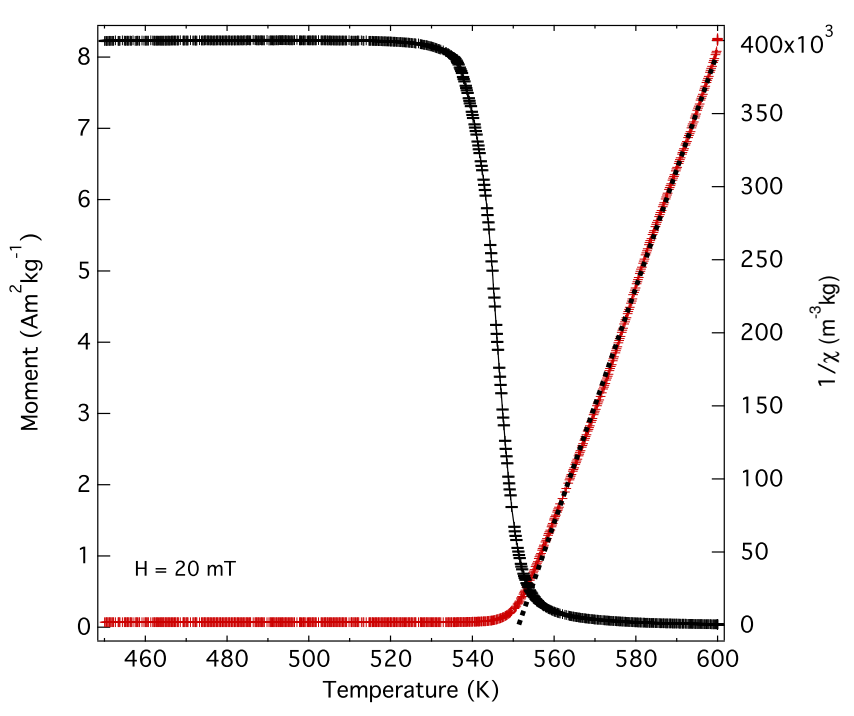

FIG. 5. MT curve (left) and inverse susceptibility (right) of ordered $\mathrm{Pd}_{3} \mathrm{Fe}$ single crystal (as used for neutron measurements) collected in $20 \mathrm{mT}$ field on warming (zero field cooled). The black dotted line represents the Curie fit to the high temperature inverse susceptibility used to determine the Curie temperature.

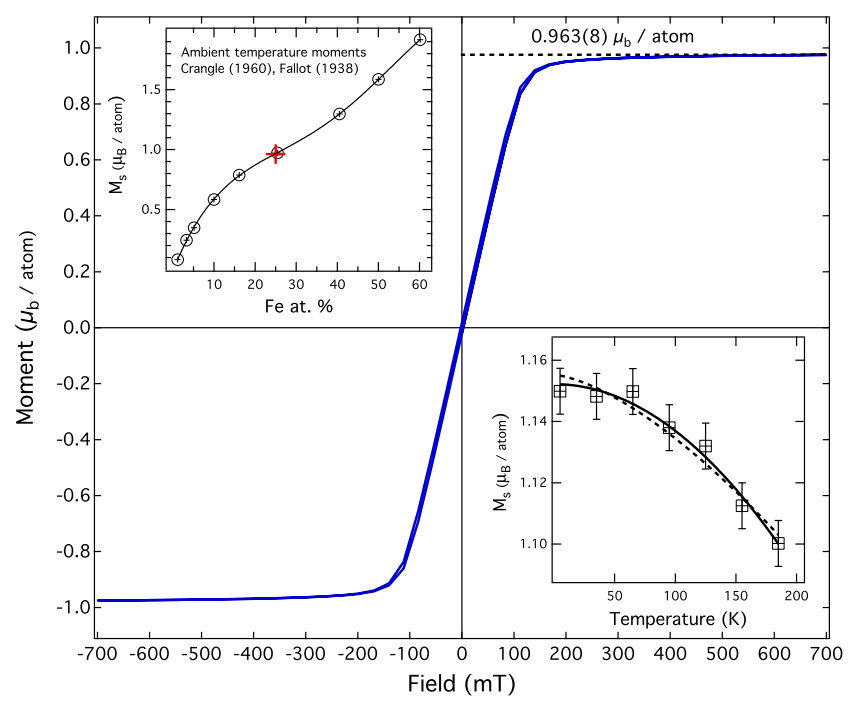

FIG. 6. Ambient temperature MH curve of single crystal $\mathrm{Pd}_{3} \mathrm{Fe}$ (as used for neutron measurements). (inset upper left) Comparison of the determined saturated moment with literature values as a function of Fe content. (+) shows the measured saturated moment for the current sample. (inset lower right) Determined saturated moment as function of temperature for $T<200 \mathrm{~K}$. Black dashed line indicates fit to $T^{3 / 2}$ expression, and the solid line a fit to a $T^{2}$ expression as described in the text.

\section{v. CONCLUSION}

We have demonstrated that there is no evidence of a large volume collapse in single or polycrystalline samples of ordered $\mathrm{Pd}_{3} \mathrm{Fe}$ up to $15 \mathrm{GPa}$. The samples were measured to be significantly (20\%) more compressible than previously re- 


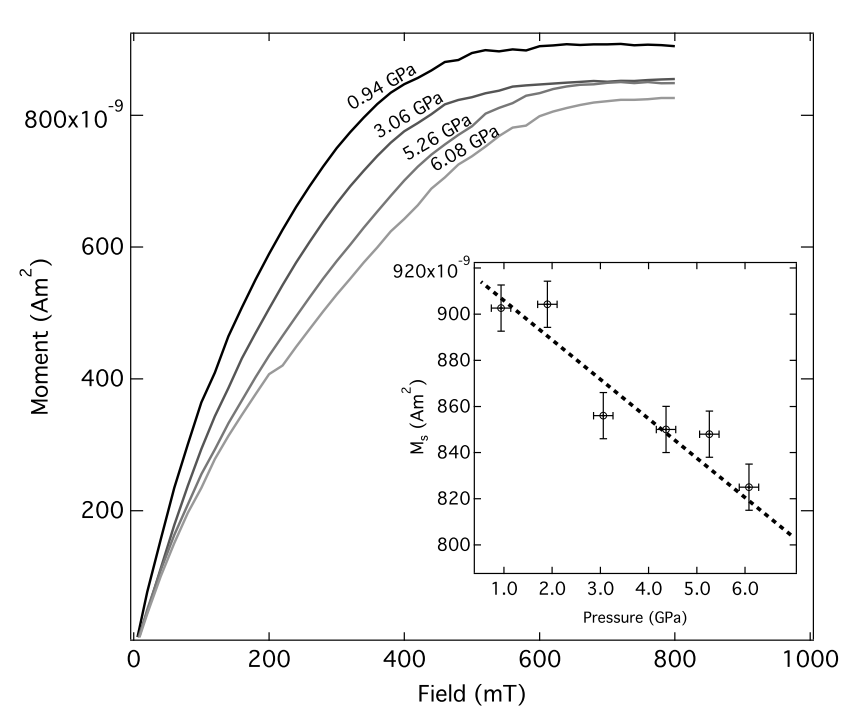

FIG. 7. Background corrected MH curves from ordered polycrystalline $\mathrm{Pd}_{3} \mathrm{Fe}$ collected at various pressures at $300 \mathrm{~K}$ in the TB$\mathrm{DAC}^{39}$. (inset) Measured change in saturated magnetic moment as a function of applied pressure with extrapolated fit to ambient pressure.

ported, with a bulk modulus of approximately $188 \mathrm{GPa}$ with fixed $B^{\prime}=4$. Analysis of the magnetic contribution to the single-crystal neutron data suggests a loss of long range order by $8 \mathrm{GPa}$, due to a pressure-driven reduction in the Curie temperature of the material. This is inconsistent with the observed transition to a non-paramagnetic state as seen in nuclear forward scattering data by Winterrose et al. ${ }^{6}$. The absence of a pressure induced Invar state is either attributed to improved hydrostaticity of the sample conditions in the present study, due to different pressure mediums being used, or due to differences in sample preparation, such as a strain induced effect due to cold rolling the sample ${ }^{6}$. In light of these measurements we suggest that further experimental data is required to determine the source of the difference between these studies. Further work is required to confirm the pressure dependence of the Curie temperature for this alloy series.

\section{SUPPLEMENTARY MATERIAL}

The online supplementary material contains a representative dataset obtained from the high-pressure single crystal measurements performed on the WISH diffractometer. There is also a representative high-pressure powder neutron diffraction pattern, and Rietveld refinement, obtained from the PEARL diffractometer. In addition, moment-temperature SQUID data performed in a higher field are shown.

\section{ACKNOWLEDGMENTS}

The authors acknowledge the Science and Technology Facilities Council for providing access to the WISH, PEARL and SXD instruments at the ISIS Neutron and Muon Source. This work was funded and supported through STFC studentship and EPSRC grant EP/J00099X. The authors wish to thank Dmitry Sokolov his assistance with preparing the single crystal samples, and Paul Steadman for his assistance with the high temperature SQUID measurements. We would also like to thank James Taylor for assisting with high temperature treatment of the precursor materials, and Daniel Nye and Gavin Stenning for their assistance with the SQUID in the Materials Characterisation Laboratory at the ISIS Neutron and Muon Source.

${ }^{1}$ J. Buschbeck, I. Opahle, S. Fähler, L. Schultz, and M. Richter, "Magnetic properties of Fe-Pd magnetic shape memory alloys: Density functional calculations and epitaxial films," Physical Review B 77, 174421 (2008).

${ }^{2}$ V. Sánchez-Alarcos, V. Recarte, J. Pérez-Landazábal, C. Gómez-Polo, V. Chernenko, and M. González, "Reversible and irreversible martensitic transformations in fe-pd and fe-pd-co alloys," The European Physical Journal-Special Topics 158, 107-112 (2008).

${ }^{3}$ H. Masumoto, H. Saitô, and T. Kobayashi, "The thermal expansion coefficients and the temperature coefficients of young's modulus of the alloys of iron and palladium," Transactions of the Japan Institute of Metals 4, 114117 (1963).

${ }^{4}$ A. Kussmann and K. Jessen, "INVAR-behaviour and magnetic moments of gamma-phase of iron-palladium alloys," Journal of the Physical Society of Japan 17, 136 (1962).

${ }^{5}$ M. Matsui, T. Shimizu, H. Yamada, and K. Adachi, "Magnetic properties and thermal expansion of Fe-Pd invar alloys," Journal of Magnetism and Magnetic Materials 15, 1201-1202 (1980).

${ }^{6}$ M. L. Winterrose, M. S. Lucas, A. F. Yue, I. Halevy, L. Mauger, J. A. Muñoz, J. Hu, M. Lerche, and B. Fultz, "Pressure-induced Invar behavior in $P d_{3} F e$," Physical Review Letters 102, 237202 (2009).

${ }^{7}$ M. Birsan, B. Fultz, and L. Anthony, "Magnetic properties of bcc Fe-Pd extended solid solutions," Physical Review B 55, 11502 (1997).

${ }^{8} \mathrm{G}$. Longworth, "Temperature dependence of the Fe $57 \mathrm{hfs}$ in the ordered alloys Fe Pd 3 and FePd near the curie temperature," Physical Review 172, 572 (1968)

${ }^{9}$ M. Matsui, H. Yamada, and K. Adachi, "A new low temperature phase (fct) of Fe-Pd invar," Journal of the Physical Society of Japan 48, 21612162 (1980).

${ }^{10}$ M. Seeger and H. Kronmüller, "The magnetic phase transition in ordered and disordered ferromagnets," Journal of magnetism and magnetic materials 78, 393-402 (1989).

${ }^{11} \mathrm{E}$. Wasserman, "Invar: Moment-volume instabilities in transition metals and alloys," Handbook of Ferromagnetic Materials 5, 237-322 (1990).

${ }^{12}$ K. Lagarec, D. Rancourt, S. Bose, B. Sanyal, and R. Dunlap, "Observation of a composition-controlled high-moment/low-moment transition in the face centered cubic Fe-Ni system: Invar effect is an expansion, not a contraction," Journal of Magnetism and Magnetic Materials 236, 107 - 130 (2001).

${ }^{13}$ R. Weiss, "The origin of the invar effect," Proceedings of the Physical Society 82, 281 (1963).

${ }^{14}$ P. Entel, E. Hoffmann, P. Mohn, K. Schwarz, and V. L. Moruzzi, "Firstprinciples calculations of the instability leading to the Invar effect," Physical Review B 47, 8706 (1993).

${ }^{15}$ P. James, O. Eriksson, B. Johansson, and I. Abrikosov, "Calculated magnetic properties of binary alloys between $\mathrm{Fe}, \mathrm{Co}, \mathrm{Ni}$, and $\mathrm{Cu}$," Physical Review B 59, 419 (1999).

${ }^{16}$ D. G. Rancourt and M.-Z. Dang, "Relation between anomalous magnetovolume behavior and magnetic frustration in invar alloys," Physical Review B 54, 12225 (1996).

${ }^{17}$ H. Akai and P. H. Dederichs, "Local moment disorder in ferromagnetic alloys," Phys. Rev. B 47, 8739-8747 (1993).

${ }^{18}$ M. van Schilfgaarde, I. Abrikosov, and B. Johansson, "Origin of the Invar effect in iron-nickel alloys," Nature 400, 46 (1999).

${ }^{19}$ L. Dubrovinsky, N. Dubrovinskaia, I. A. Abrikosov, M. Vennström, F. Westman, S. Carlson, M. van Schilfgaarde, and B. Johansson, "Pressureinduced invar effect in fe-ni alloys," Physical Review Letters 86, 4851 (2001). 
${ }^{20}$ W. G. Stirling and R. A. Cowley, "Spin waves in Pd3Fe," Solid State Communications 11, 271-274 (1972).

${ }^{21}$ M. Fallot, "Les alliages du fer avec les métaux de la famille du platine," in Annales de Physique, Vol. 11 (1938) pp. 291-332.

${ }^{22}$ B. Dutta, S. Bhandary, S. Ghosh, and B. Sanyal, "First-principles study of magnetism in $\mathrm{Pd}_{3} \mathrm{Fe}$ under pressure," Physical Review B 86, 024419 (2012).

${ }^{23}$ Y. O. Kvashnin, S. Khmelevskyi, J. Kudrnovskỳ, A. N. Yaresko, L. Genovese, and P. Bruno, "Noncollinear magnetic ordering in compressed $\mathrm{FePd}_{3}$ ordered alloy: A first principles study," Physical Review B 86, 174429 (2012).

${ }^{24}$ G. Ghosh, C. Kantner, and G. Olson, "Thermodynamic modeling of the Pd$\mathrm{X}(\mathrm{X}=\mathrm{Ag}, \mathrm{Co}, \mathrm{Fe}, \mathrm{Ni})$ systems," Journal of Phase Equilibria and Diffusion 20, 295 (1999).

${ }^{25}$ E. Raub, H. Beeskow, and O. Loebich, "Das zustandsbild eisen-palladium unterhalb 950-degrees-c," Zeitschrift Fur Metallkunde 54, 549-552 (1963).

${ }^{26} \mathrm{P}$. N. Stetsenko and Y. I. Avksentev, "Hyperfine interactions in an ordering Pd3Fe alloy," Soviet Physics - JETP 33, 961-962 (1971).

${ }^{27} \mathrm{~J}$. Czochralski, "Ein neues verfahren zur messung der kristallisationsgeschwindigkeit der metalle," Zeitschrift für Physikalische Chemie 92, 219-221 (1918).

${ }^{28}$ W. Whitley, C. Stock, and A. D. Huxley, "A laboratory-based Laue X-ray diffraction system for enhanced imaging range and surface grain mapping," Journal of Applied Crystallography 48, 1342-1345 (2015).

${ }^{29}$ D. A. Keen, M. J. Gutmann, and C. C. Wilson, "SXD-the single-crystal diffractometer at the isis spallation neutron source," Journal of Applied Crystallography 39, 714-722 (2006).

${ }^{30}$ M. Gutmann, "SXD2001," ISIS Facility, Rutherford Appleton Laboratory, Oxfordshire, England (2005).

${ }^{31}$ M. J. Gutmann, "A 3D profile function suitable for integration of neutron time-of-flight single crystal diffraction peaks," Nuclear Instruments and Methods in Physics Research Section A: Accelerators, Spectrometers, Detectors and Associated Equipment 848, 170 - 173 (2017).

${ }^{32}$ G. M. Sheldrick, "A short history of SHELX," Acta Crystallographica Section A 64, 112-122 (2008).

${ }^{33}$ L. C. Chapon, P. Manuel, P. G. Radaelli, C. Benson, L. Perrott, S. Ansell, N. J. Rhodes, D. Raspino, D. Duxbury, E. Spill, et al., "WISH: the new powder and single crystal magnetic diffractometer on the second target station," Neutron News 22, 22-25 (2011).

${ }^{34}$ C. L. Bull, N. P. Funnell, M. G. Tucker, S. Hull, D. J. Francis, and W. G. Marshall, "PEARL: the high pressure neutron powder diffractometer at ISIS," High Pressure Research 36, 493-511 (2016).

${ }^{35}$ S. Klotz, J. M. Besson, G. Hamel, R. J. Nelmes, J. S. Loveday, W. G. Marshall, and R. M. Wilson, "Neutron powder diffraction at pressures beyond 25 GPa," Applied Physics Letters 66, 1735-1737 (1995).

${ }^{36}$ S. Klotz, J.-C. Chervin, P. Munsch, and G. L. Marchand, "Hydrostatic limits of 11 pressure transmitting media," Journal of Physics D: Applied Physics 42, 075413 (2009).

${ }^{37}$ T. Strässle, S. Klotz, K. Kunc, V. Pomjakushin, and J. S. White, "Equation of state of lead from high-pressure neutron diffraction up to $8.9 \mathrm{GPa}$ and its implication for the $\mathrm{NaCl}$ pressure scale," Physical Review B 90, 014101 (2014).

${ }^{38}$ C. L. Bull, M. Guthrie, R. J. Nelmes, J. S. Loveday, K. Komatsu, H. Hamidov, and M. J. Gutmann, "Time-of-flight single-crystal neutron diffraction to $10 \mathrm{GPa}$ and above," High Pressure Research 29, 780-791 (2009).

${ }^{39}$ G. Giriat, W. Wang, J. P. Attfield, A. D. Huxley, and K. V. Kamenev, "Turnbuckle diamond anvil cell for high-pressure measurements in a superconducting quantum interference device magnetometer," Review of Scientific Instruments 81, 073905 (2010).
${ }^{40}$ A. Dewaele, M. Torrent, P. Loubeyre, and M. Mezouar, "Compression curves of transition metals in the mbar range: Experiments and projector augmented-wave calculations," Physical Review B 78, 104102 (2008).

${ }^{41}$ R. C. Wayne and L. C. Bartel, "Pressure dependence of the Curie temperatures of the fcc alloys of Fe with Ni, Pd and Pt," Physics Letters A 28 , 196-197 (1968).

${ }^{42}$ S. Wei, R. Duraj, R. Zach, M. Matsushita, A. Takahashi, H. Inoue, F. Ono, H. Maeta, A. Iwase, and S. Endo, "The effect of pressure on the Curie temperature in Fe-Ni Invar mechanical alloys," Journal of Physics: Condensed Matter 14, 11081 (2002).

${ }^{43}$ P. Vinet, J. Ferrante, J. H. Rose, and J. R. Smith, "Compressibility of solids," Journal of Geophysical Research: Solid Earth 92, 9319-9325 (1987).

${ }^{44}$ J. W. Cable, E. O. Wollan, W. C. Koehler, and M. K. Wilkinson, "Neutron diffraction investigations of ferromagnetic palladium and iron group alloys," Journal of Applied Physics 33, 1340-1340 (1962).

${ }^{45}$ C. J. Ridley and K. V. Kamenev, "High pressure neutron and X-ray diffraction at low temperatures," Zeitschrift für Kristallographie-Crystalline Materials 229, 171-199 (2014).

${ }^{46}$ C. A. Kuhnen and E. Z. Da Silva, "Magnetic properties and calculated electronic structure of iron-palladium alloys," Physical Review B 46, 8915 (1992).

${ }^{47}$ M. De Jong, W. Chen, T. Angsten, A. Jain, R. Notestine, A. Gamst, M. Sluiter, C. K. Ande, S. Van Der Zwaag, J. J. Plata, et al., "Charting the complete elastic properties of inorganic crystalline compounds," Scientific Data 2, 150009 (2015).

${ }^{48}$ J. Rodriguez-Carvajal, "FULLPROF: a program for rietveld refinement and pattern matching analysis," in Satellite Meeting on Powder Diffraction of the XV congress of the IUCr, Vol. 127 (Toulouse, France:[sn], 1990).

${ }^{49}$ K. M. Dąbrowski, D. T. Dul, T. Jaworska-Gołąb, J. Rysz, and P. Korecki, "X-ray fluorescence holography studies for a Cu3Au crystal," Nuclear Instruments and Methods in Physics Research Section B: Beam Interactions with Materials and Atoms 364, 136-141 (2015).

${ }^{50} \mathrm{Q}$. Zeng and I. Baker, "The effects of local versus bulk disorder on the magnetic behavior of stoichiometric Ni3Al," Intermetallics 15, 419-427 (2007).

${ }^{51} \mathrm{H}$. Yasuda, T. Takasugi, and M. Koiwa, "Elasticity of Ni-based L12-type intermetallic compounds," Acta Metallurgica et Materialia 40, 381-387 (1992).

${ }^{52}$ J. E. Goldman and R. Smoluchowski, "Magnetostriction and orderdisorder," Physical Review 75, 140 (1949).

${ }^{53}$ T. Nautiyal and S. Auluck, "The electronic structure and magnetism of MoPd3 and MnPd3," Journal of Physics: Condensed Matter 1, 2211 (1989).

${ }^{54} \mathrm{C}$. $\mathrm{Li}$ and $\mathrm{P}$. Wu, "Correlation of bulk modulus and the constituent element properties of binary intermetallic compounds," Chemistry of Materials 13, 4642-4648 (2001).

${ }^{55}$ R. J. Angel, M. Bujak, J. Zhao, G. D. Gatta, and S. D. Jacobsen, "Effective hydrostatic limits of pressure media for high-pressure crystallographic studies," Journal of Applied Crystallography 40, 26-32 (2007).

${ }^{56}$ J. C. Chervin, B. Canny, J. M. Besson, and P. Pruzan, "A diamond anvil cell for IR microspectroscopy," Review of Scientific Instruments 66, 25952598 (1995).

${ }^{57}$ J. Ruiz-Fuertes, D. Errandonea, R. Lacomba-Perales, A. Segura, J. González, F. Rodríguez, F. Manjón, S. Ray, P. Rodríguez-Hernández, A. Muñoz, et al., "High-pressure structural phase transitions in CuWO4," Physical Review B 81, 224115 (2010).

${ }^{58}$ J. Crangle, "Ferromagnetism in Pd-rich palladium-iron alloys," Philosophical Magazine 5, 335-342 (1960).

${ }^{59}$ S.-H. Fang, "Spontaneous magnetization of palladium iron alloys," Chinese Journal of Physics 5, 55-62 (1967). 